\title{
Porosity Formation and Meltpool Geometry Analysis Using High-speed, in situ Imaging of Directed Energy Deposition
}

\author{
Samantha Webster ${ }^{1 *}$, Sarah Wolff ${ }^{2}$, Jennifer Bennett ${ }^{1}$, Tao Sun ${ }^{3}$, Jian Cao ${ }^{1}$ and Kornel Ehmann ${ }^{1}$ \\ 1. Mechanical Engineering, Northwestern University, Evanston, IL, USA. \\ 2. Applied Materials Division, Argonne National Laboratory, Lemont, IL, USA. \\ 3. X-ray Science Division, Argonne National Laboratory, Lemont, IL, USA. \\ * Corresponding author: samanthawebster2021@u.northwestern.edu
}

While directed energy deposition (DED) can provide tremendous flexibility in both geometry and materials, there are still many challenges that need to be overcome before this technology can be freely used in industry ${ }^{1}$. Previous work has established a link between the shape of pores and cooling rate ${ }^{2}$, however, a more fundamental understanding of this process still needs to be fully established. Highspeed synchrotron imaging can provide adequate spatial and temporal resolutions to capture critical phenomena such as melt pool dynamics, powder entrainment, and defect formation ${ }^{3-5}$. In this study, the high-speed synchrotron X-ray imaging technique at the Advanced Photon Source (APS) is used to observe dynamic phenomena involved in the DED process. Different from previous publications, this work was carried out using an innovative home-built high-throughput DED apparatus, where a large range of process parameters could be effectively studied without the need of constantly opening the chamber to load new samples and spending time re-aligning them. This largely increased the experiment efficiency. The DED setup consists of an aluminum enclosure, a DMG single powder nozzle, a motorized sample drum, a $500 \mathrm{~W}$ continuous wave ytterbium fiber laser, and a fast motorized stage for deposition. The enclosure was filled with Argon during experiments to prevent excessive oxidation. The substrates to be deposited on were $50 \mathrm{~mm}$ by $10 \mathrm{~mm}$ thin rectangle plates cut from $406 \mu \mathrm{m}$ thick sheets of Grade 5 Titanium alloy (Ti-6Al-4V). The powder used in deposition was 45-150 $\mu \mathrm{m}$ Ti-6Al-4V, and Argon carrier/shield gas was used in the nozzle. Ti-6Al-4V was chosen because it is one of most commonly used materials in metal AM and has many important applications in the fields of aerospace and aviation. The parameters in this study can be combined to define the 'effective global energy density', GED' $=\mathrm{P} /(\mathrm{PF} \cdot \mathrm{V} \cdot \mathrm{A})$, where $\mathrm{P}$ is the laser power, $\mathrm{PF}$ is the powder flow rate, $\mathrm{V}$ is the scan speed, and $\mathrm{A}$ is the beam area. GED' represents the ratio of energy to mass that is added to the sample and is an effective way to describe DED from an input parameter perspective. Since the power density of the laser used in the experiment is higher than that in a commercial DED process, the scan speeds chosen were also faster. In the experiment, the beamline components and the DED system were controlled collectively using a series of delay generators. A typical procedure is: X-ray shutters open, powder nozzle turns on, deposition stage accelerates to steady state, imaging camera turns on, fiber laser turns on and melts the powder in the X-ray window, X-ray shutters close. One example of parameters used in the experiments are: $300 \mathrm{~W}$ laser power, $500 \mathrm{mg} / \mathrm{s}$ powder flow rate, and $100 \mathrm{~mm} / \mathrm{s}$ scan speed. Images were taken at $80,000 \mathrm{fps}$ in a $2 \mathrm{~mm} \times 2 \mathrm{~mm}$ window.

Observed phenomena include pore formation, melt pool geometry and fluctuations, and powder particle capture. Representative X-ray images are shown in Fig. 1. Here, the generation of pores in the melt pool can be seen in Fig. 1 (right panel), which corresponds to a higher GED' or larger energy input. Pores in AM parts could be detrimental, as they largely reduced the fatigue life and corrosion resistance; however, they could also be generated deliberately to control some other properties of the parts in a positive way, such as tuning the thermal conductivity. The in situ X-ray imaging experiment can help 
increase understanding of the porosity formation mechanism in the DED process, and the quantitative structure information will be very valuable for improving current numerical models. Melt pool length and depth measurements were also taken, and the combined aspect ratio is reported in Fig. 2. It was traditionally believed that a larger energy input would create a deeper melt pool and a faster scan speed would lead to a longer melt pool. However, neither of those trends is observed here. In conclusion, the high-speed synchrotron X-ray imaging technique allows high spatial and temporal observations of the highly dynamic laser DED process. It was found that energy input linearly increases the number of pores formed, and that melt pool geometry may not be accurately described by GED'. Future work will include developing automatic melt pool measurement methods, determining the major pore generation and elimination mechanism, and observing the mechanics and quantity of particle capture. In all, understanding the physics behind defect generation and powder capture will not only lead to a better process for high-quality AM parts, but also facilitate the development of high-fidelity models for AM process and material design [6].

References:

[1] T Debroy, L Wei and JS Zuback, Progress in Materials Science 92 (2018), p. 112.

[2] S Wolff, S Lin and EJ Faierson, Acta Materialia 132 (2017) p. 106.

[3] S Wolff, H Wu and ND Parab, Scientific Reports 9 (2019), p. 962.

[4] ND Parab, C Zhao and R Cunningham, Journal of Synchrotron Radiation 25 (2018), p. 1467.

[5] S Ly et al., Scientific Reports 7 (2017), p. 4085.

[6] The author acknowledges Niranjan Prab for his help at the APS beamline, and Yi Shi, Nicolas Prieto, Haiguang Liao, David Pritchet, Yanjie Yuan, and Marco Giovanni for their work on the set-up.
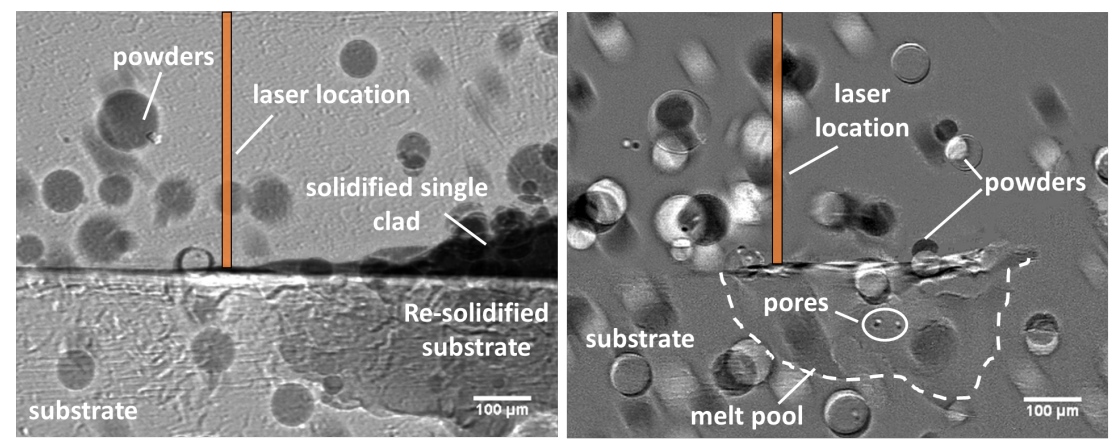

Figure 1. High- speed synchrotron X-ray images of the DED process. (Left) Background-subtracted image showing in situ build process. (Right) Subsequent images divided to show melt pool boundary and particle movement.
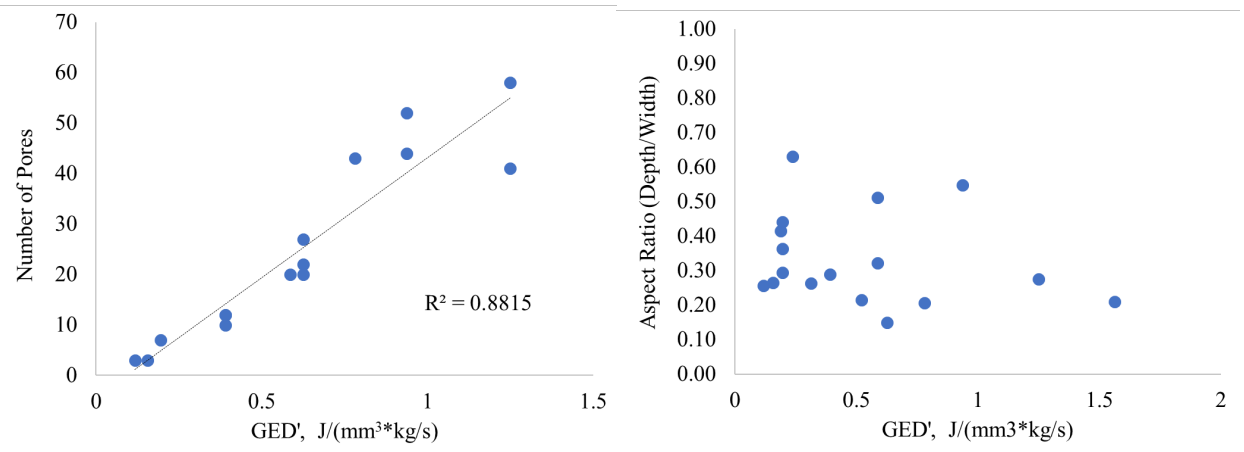

Figure 2. Number of generated pores (left) and meltpool aspect ratio (right) vs. global energy density. The number of pores increases linearly at low energy densities. The aspect ratio ranges from 0.2-0.7. 